\title{
Pain in individuals who suffered a spinal cord injury. How does pain interfere. Hope for improved management
}

\author{
JJ Wyndaele, Editor \\ Antwerp University Hospital, Antwerp, Belgium \\ E-mail: spinalcord@uza.be
}

Dear Spinal Cord reader,

Pain can be an extreme burden in patients, and the fact that pain interferes with rehabilitation, performance and quality of life is a well-known phenomenon in spinal cord injury (SCI) management and follow-up. An important review on the management of neuropathic pain following spinal cord injury by Phillip Siddall was published in Spinal Cord last year (ISCoS Guttmann lecture, 2008). It provides an overview of our current understanding of the problem of neuropathic pain following SCI and suggests possible therapeutic options for the near future. The author states that substantial progress has been made in identifying the common types of pain that occur following SCI; in determining the prevalence and characteristics of pain; in investigating some of the pathophysiological changes in the nervous system that may contribute to the presence of neuropathic SCI pain; and in examining the effectiveness of some treatments. Major challenges, however, remain. There is still need to reach consensus on an SCI pain taxonomy. The understanding of mechanisms and the relative contribution of changes in the periphery, spinal cord and brain is incompletely understood. There are few studies that indicate effective treatment options, particularly for neuropathic SCI pain. Treatment of the biological and psychological contributors to pain is also often fragmented. Recent studies suggest the potential usefulness of new treatment approaches, such as selective pharmacological agents, application of novel neurostimulation techniques and the use of cognitive approaches to modify the pain experience. Siddall concludes that increasing understanding of the problem combined with the promise of these new approaches offers hope for improved management of neuropathic pain following SCI in the near future.

Eva Widerström-Noga and colleagues remind us that pain interference is an important outcome measure in clinical pain trials. However, after SCI, interference caused by pain may be difficult to separate from interference caused by the physical impairment. Their study shows that the Life Interference subscale of the Multidimensional Pain Inventory, SCI-version (MPI-SCI), administered in an interview format, is appropriate for measuring pain-specific interference in the SCI chronic pain population. Other interesting papers on pain can be found in the January 2008 issue of Spinal Cord.

A case report by Calder et al. described the overlooked and/or under-reported incidence of acute neurogenic pulmonary edema associated with episodes of dysreflexia in the SCI population.

It is important to focus knowledge and improve communication on spinal cord care around the world. In the December 2008 issue, several International SCI Data Sets and International Standards were published. The International Urinary Tract Imaging Basic Spinal Cord Injury Data Set is published here.

Many other interesting contributions can be found in this, the May issue of Spinal Cord. Enjoy them. 